\title{
Association between hepatitis B virus infection and diabetes mellitus: A meta-analysis
}

\author{
CUIXIA CAI, JUN ZENG, HUIHUI WU, RONG SHI, MIN WEI, YUAN GAO and WENLI MA \\ Institute of Genetic Engineering, Southern Medical University, Guangzhou, Guangdong 510515, P.R. China
}

Received August 1, 2014; Accepted April 23, 2015

DOI: $10.3892 /$ etm.2015.2537

\begin{abstract}
Hepatitis B virus (HBV) infection has been shown by certain studies to be associated with diabetes mellitus (DM); however, the results of these studies were controversial. For that reason, a meta-analysis of the literature was performed in order to determine the association between HBV infection and the prevalence of DM more accurately. The PubMed, Embase, Chinese National Knowledge Infrastructure and Wan Fang databases, as well as the Chinese Science and Technology Journal Database, were searched for literature published until June 2014. The reference lists of all relevant articles were also searched. The summary odds ratios (ORs) and their corresponding $95 \%$ confidence intervals (95\% CIs) were calculated based on a random-effects model. Heterogeneity was assessed using the $\mathrm{I}^{2}$ statistic. Subgroup analyses were conducted based on study type and region for the purpose of assessing the factors that could potentially affect the outcome. A total of 15 eligible studies (in 14 articles) were selected for the meta-analysis, involving 12,974,690 HBV-infected patients and 231,776,232 controls. The OR for the prevalence of DM was $1.33(95 \% \mathrm{CI}, 1.09-1.62 ; \mathrm{P}=0.005)$ between the patients with HBV infection and the controls. The subgroup analysis based on study type revealed a significantly higher prevalence of DM in the HBV-infected group than that in the control group in both case-control (OR, 1.89; 95\% CI, 1.08-3.30; $\mathrm{P}=0.025)$ and cross-sectional (OR, 1.41; 95\% CI, 1.04-1.90; $\mathrm{P}=0.027$ ) studies. The subgroup analysis based on region revealed a significantly higher prevalence of DM in the HBV-infected group than in the control group in the Asia-Pacific region (OR, 1.67; 95\% CI, 1.08-2.58; $\mathrm{P}=0.022)$. Compared with uninfected patients, the pooled results suggest that $\mathrm{HBV}$-infected patients have a higher risk of developing DM; however, given the fact that this is a meta-analysis of observational studies, further
\end{abstract}

Correspondence to: Dr Wenli Ma and Dr Yuan Gao, Institute of Genetic Engineering, Southern Medical University, 1838 North Guangzhou Avenue, Guangzhou, Guangdong 510515, P.R. China E-mail: wenli668@gmail.com

E-mail: gaoyuan1623@163.com

Key words: hepatitis B virus, diabetes mellitus, meta-analysis randomized controlled trials are required in order to reach a more accurate conclusion.

\section{Introduction}

Diabetes mellitus (DM) is a disease with one of the fastest growing incidences worldwide. By 2030 developing countries will face an increase of $69 \%$ and industrialized countries of $20 \%$ of the number of patients with diabetes compared with 2010. For Africa $>18$ million, according to some estimations even 24 million, diabetic patients are predicted for the year 2030 (1). It has recently been suggested that diabetes increases the risk of a variety of cancers, including breast, pancreatic, colorectal and kidney cancer (2). Hepatitis B virus (HBV) infection is one of the most severe infections and constitutes a major risk factor for mortality from cirrhosis and liver cancer (3). A number of studies have identified an association between $\mathrm{HBV}$ infection and the prevalence of DM; however, the results of those studies were inconclusive. Certain studies have supported the increased risk of DM in HBV-infected patients when compared with non-HBV-infected controls (4-9) and certain studies have had different results (10-17). To the best of our knowledge, no meta-analysis has ever focused on assessing the association between HBV infection and the risk of DM. The present meta-analysis was conducted in order to gain an enhanced understanding of the association between the conditions.

\section{Materials and methods}

Identification and eligibility of relevant studies. The PubMed, Embase, Chinese National Knowledge Infrastructure and Wan Fang databases, as well as the Chinese Science and Technology Journal Database, were used to perform a comprehensive literature search for relevant articles published until June 2014. The following keywords were used: 'Hepatitis B virus' or ' $\mathrm{HBV}$ ' or 'hepatitis B' and 'diabetes', 'diabetes mellitus' or 'DM'. The search was limited to human studies and publication in either English or Chinese. The literature search was conducted independently by two reviewers. An additional manual search of the reference lists of all relevant articles for all available review articles and primary studies was also performed.

Study selection criteria. The inclusion criteria for this study were as follows: i) Evaluation of the association between 
HBV infection and the risk of DM; ii) presence of at least one comparison group without $\mathrm{HBV}$ and hepatitis $\mathrm{C}$ virus (HCV) infection; iii) studies with accessible full texts and iv) DM confirmed based on a) self-reported DM (i.e., diagnosed by a physician), b) fasting plasma glucose levels $>7.0 \mathrm{mmol} / \mathrm{l}$ on two separate occasions or c) impaired fasting glycemia of 6.1-7.0 mmol/1 without insulin medication. Where available, hepatitis B surface antigen (HBsAg) and/or antibody against hepatitis B core antigen (anti-HBc) and/or HBV DNA were detected to confirm HBV infection.

The exclusion criteria for this study were as follows: i) Gestational DM; ii) observational studies without control groups and case reports; iii) subset of a published article with the same data and by the same authors; and iv) studies involving patients with chronic liver disease with alternative etiologies, such as autoimmune hepatitis, steatohepatitis, cirrhosis, primary biliary cirrhosis, hepatocellular carcinoma and primary cholangitis.

Data extraction. The necessary information was extracted from all eligible studies by two independent investigators, according to the aforementioned inclusion criteria. Disagreements were resolved by consensus or with the assistance of a third reviewer. The following information was collected: i) First author's name, ii) year of publication, iii) country of origin, iv) age of the patients, v) HBV detection method, vi) reported odds ratio (OR) with 95\% confidence interval (CI) and vi) number of cases and controls.

Statistical analysis. The fixed-effect or random-effects model was selected as appropriate, depending on the heterogeneity among the studies included in the present meta-analysis. The degree of heterogeneity among the studies was assessed using the $\mathrm{I}^{2}$ test. An $\mathrm{I}^{2}$ value $>50 \%$ was considered to represent substantial heterogeneity. The assumption that the OR in a case-control study approximates the relative risk in a cohort study was used. The fixed-effect model was used in the absence of significant heterogeneity $\left(\mathrm{I}^{2}<50 \%\right)$, whereas the random-effects model was selected in the presence of significant heterogeneity $\left(\mathrm{I}^{2}>50 \%\right) . \mathrm{P}<0.05$ was considered to indicate a statistically significant difference for all included studies. Subgroup analyses were performed based on study types and ethnic groups. Begg's funnel plot and Egger's weighted regression method were used to test for publication bias. All analyses listed above were conducted using Stata software (version 12.0; StataCorp LP, College Station, TX, USA).

\section{Results}

Description of studies. As summarized in Table I, 14 articles (4-17), which were published between 2000 and 2013 and included 15 independent studies with 12,974,690 HBV-infected patients and 231,776,232 controls in total, were assessed in the present meta-analysis. Among these 15 studies, 8 were cross-sectional studies, 5 were case-control studies and 2 were cohort studies. Six studies showed a significantly higher prevalence of DM in HBV-infected patients than in the controls (4-9), 7 studies lacked evidence of a significant association between DM and HBV infection $(6,12-17)$ and 2 studies revealed a lower prevalence of DM in patients with $\operatorname{HBV}(10,11)$. The 15 studies involved 244,750,922 attendees and reported a total DM prevalence of $7.40 \%(18,118,293 / 244,750,922)$. The cumulative sample size of HBV-infected patients was $12,974,690$, of which 1,064,171 also suffered from DM (8.20\%). Out of the $231,776,232$ controls, 17,054,122 had DM (7.36\%). The OR for the prevalence of DM in the patients with HBV infection was 1.33 (95\% CI, 1.09-1.62; $\mathrm{P}=0.005)$, when compared with the controls (Fig. 1).

Subgroup analysis. The results of the present meta-analysis demonstrated that the HBV-infected patients had a higher risk of developing DM when compared with the uninfected patients (OR, 1.33; 95\% CI, 1.09-1.62; $\mathrm{P}=0.005$ ); however, the heterogeneity between the two groups was high $\left(\mathrm{I}^{2}=93.8 \%\right)$. Subgroup analyses based on study type and region were performed to investigate the factors that could impact the overall results. The results of the study type-based subgroup analysis indicated that the prevalence of DM in the HBV group was significantly higher than that in the control group, both in case-control (11.45 vs. 9.30\%; OR, 1.89; 95\% CI, 1.08-3.30; $\mathrm{P}=0.025)$ and cross-sectional (8.20 vs. 7.36\%; OR, 1.40; 95\% CI, 1.04-1.90; $\mathrm{P}=0.027$ ) studies; however, the prevalence of DM in the case group was lower than that in the control group in the cohort studies (9.40 vs. 11.87\%; OR, 0.77; 95\% CI, 0.71-0.84; $\mathrm{P}<0.001$ ) (Fig. 1). The results of the region-based subgroup analysis showed that the prevalence of DM in the HBV group was significantly higher than that in the control group in the Asia-Pacific region (OR, 1.67; 95\% CI, 1.08-2.58; $\mathrm{P}=0.022$ ); however, no significant difference was identified between the two groups in the USA (OR, 0.97; 95\% CI, 0.73-1.30; $\mathrm{P}=0.86$ ) (Fig. 2).

Publication bias. Funnel plot analysis did not reveal any evidence of publication bias in the 15 studies [Begg's test $\mathrm{z}=0.99$ (continuity corrected); $\mathrm{P}=0.322$ ] (Fig. 3). The Egger's test also indicated a lack of significant publication bias $(\mathrm{P}=0.906)$.

\section{Discussion}

Liver disease of various etiologies, including HCV infection, has been implicated as a cause of DM in several previous studies (18-20). A hypothesis that there may be an association between HBV infection and DM incidence has been proposed $(8,9)$; however, the results remain controversial. In order to resolve this controversy, 15 studies were selected for the present meta-analysis, the aim of which was to comprehensively evaluate the association between $\mathrm{HBV}$ infection and the prevalence of DM.

The present study indicates that patients with HBV infection are at higher risk of developing DM when compared with patients without HBV infection. The findings of this study are in accordance with those of a large-sample, cross-sectional study in the USA (5). According to the aforementioned results, HBV infection could comprise a potential risk factor for the development of DM. Several mechanisms may be involved in the association between HBV infection and the prevalence of DM. First, the liver is an organ that plays a key role in the regulation of glucose homeostasis by 


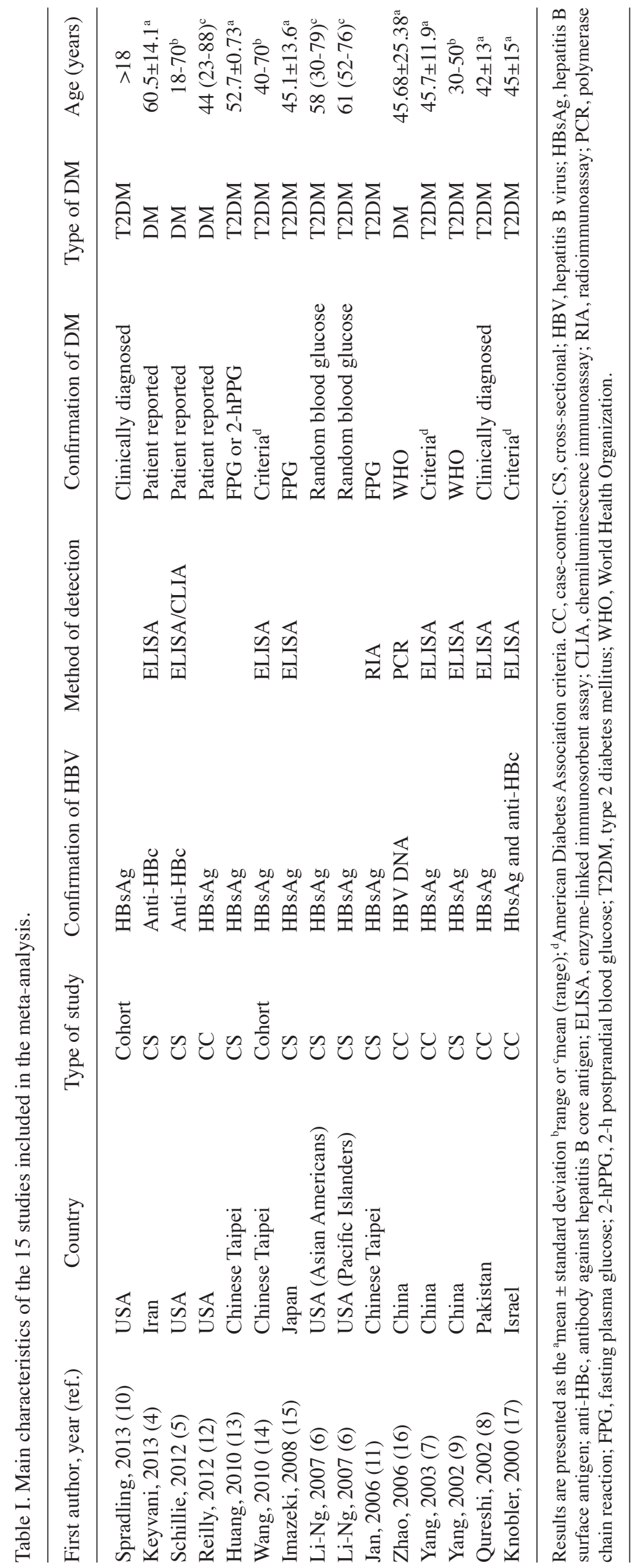




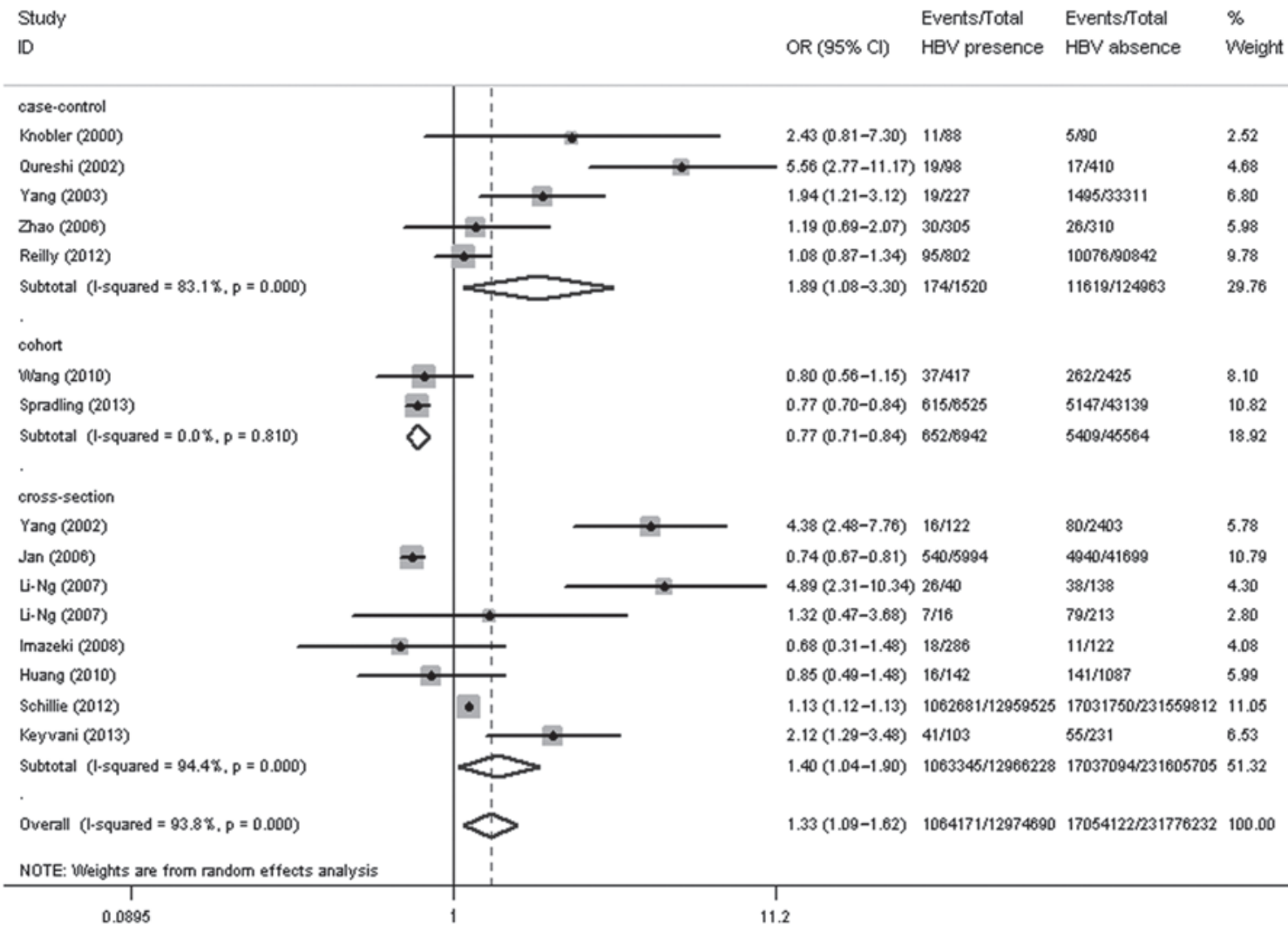

Figure 1. Study type-based subgroup analysis on the prevalence of diabetes mellitus in the HBV infection group compared with the control group. HBV, hepatitis B virus; OR, odds ratio; CI, confidence intervals.

\begin{tabular}{l} 
Study \\
ID \\
\hline Asia \& Pacific \\
Knobler (2000) \\
Qureshi (2002) \\
Yang (2002) \\
Yang (2003) \\
Zhao (2006) \\
Jan (2006) \\
Li-Ng (2007)
\end{tabular}

Figure 2. Region-based subgroup analysis on the prevalence of diabetes mellitus in the HBV infection group compared with the control group. HBV, hepatitis B virus; $\mathrm{OR}$, odds ratio; $\mathrm{CI}$, confidence intervals.

balancing the storage and output of glucose. Liver damage caused by HBV infection may lead to a glycometabolism disorder $(21,22)$, and persistent inflammatory activities in the liver may cause defective glucose homeostasis. Inflammatory mediators, such as tumor necrosis factor- $\alpha$ and nitric oxide, have been shown to impair the metabolic action of insulin in the liver, which results in hepatic dysfunction and, in turn, leads to insulin resistance (23-26). Furthermore, 


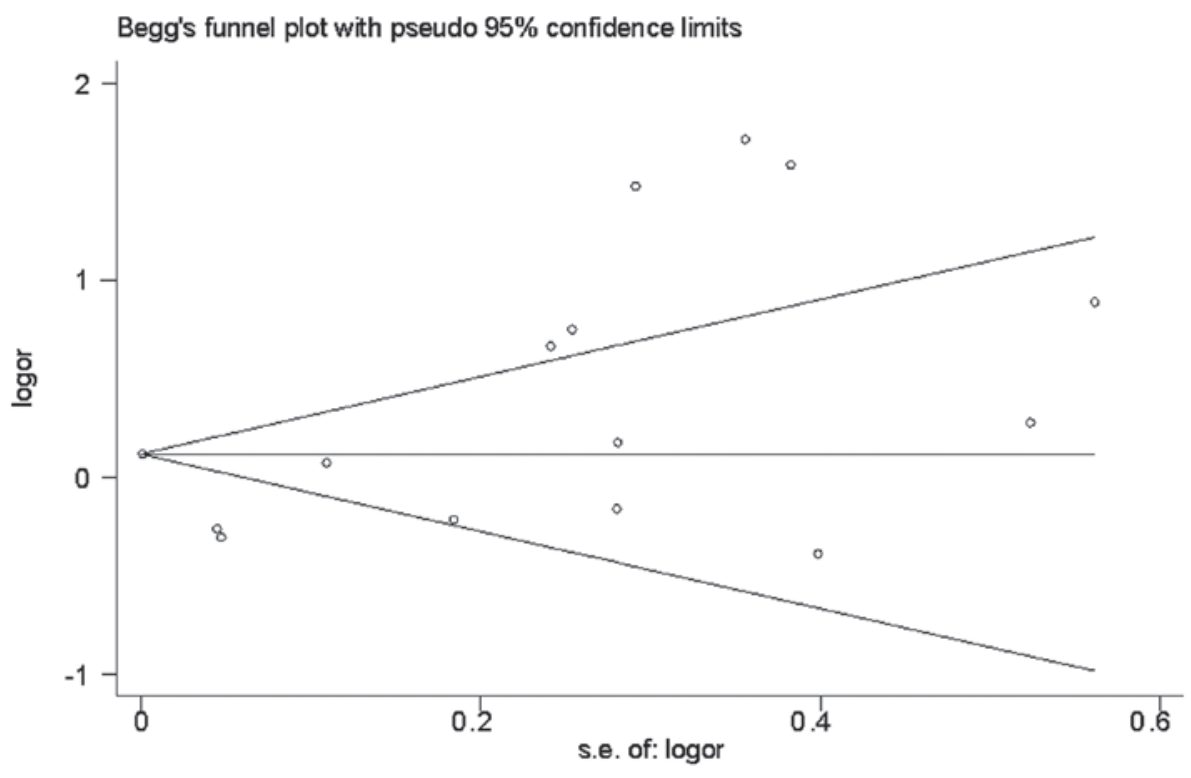

Figure 3. Estimating publication bias using Begg's test. OR, odds ratio; SE, standard error.

inducible nitric oxide synthase expression has been shown to be elevated in the liver of patients suffering from chronic HBV infection (27). Secondly, several studies $(28,29)$ have found HBV infection in the pancreas. The replication of HBV in extrahepatic sites, such as the pancreas, is responsible for $\beta$-cell damage and may ultimately lead to diabetes $(28,29)$. In addition, insulin resistance may be involved in the pathogenesis of hepatogenous diabetes. Ji et al (30) reported that the pre-S2 protein of HBV decreased the expression of the insulin receptor gene, leading to insulin resistance.

In the present meta-analysis, it was notable that the subgroup analysis showed the prevalence of DM in the HBV group to be significantly higher than that in the control group, both in the case-control and cross-sectional studies; however, the prevalence of DM in the case group was lower than that in the control group in the cohort studies, which included a study performed on subjects from the USA. Region-based subgroup analysis revealed that HBV infection was associated with an increased risk of DM in the Asia-Pacific region, while no significant difference was found in the prevalence of DM between the HBV-infected patients and the controls in the USA. The low incidence of $\mathrm{HBV}$ infection and high prevalence rate of DM in the USA may explain these results. The overall prevalence of chronic HBV infection in the USA is $0.4 \%$, whereas $10-15 \%$ of Asian Americans/Pacific Islanders suffer from chronic HBV infection. In most Asian regions, the prevalence of HBV infection is $>8.0 \%$ (29). By comparison, $\sim 12.4 \%$ of the US population had been diagnosed with DM up to 2010 (31), while the prevalence of DM in Asia has been reported to be $8.7 \%$ (32).

Of note, the Keelung Community-Based Integrated Screening Study (14), conducted in Chinese Taipei, found that the DM prevalence was lower among HBV-infected patients than that among uninfected patients. Two more studies $(11,12)$ from Chinese Taipei also showed a lower prevalence of DM among HBV-infected patients, although the differences were not statistically significant. The reason for this finding is unclear but may be due to dietary, genetic or other environmental differences among these ethnic groups.
Despite the considerable efforts made and resources employed to test the association between HBV infection and the DM prevalence, the present meta-analysis had certain limitations. First, HBV infection was diagnosed by different serum markers across the included studies. The markers included the HBsAg, anti-HBc, HBV DNA or a combination of more than one serum marker, making it impossible to distinguish between past and the ongoing HBV infections. Furthermore, the severity of the hepatitis was not known, since only a few studies had reported the aminotransferase levels. Secondly, three different types of studies were involved in the present meta-analysis: Case-control, cross-sectional and cohort studies. The various types of study designs could have been partially responsible for the heterogeneity across the studies. In addition, the majority of the studies included were retrospective, making them susceptible to recall and selection bias. No evidence for an association between HBV infection and the prevalence of DM was provided by the 2 cohort studies; however, the results should be considered with caution since the number of patients included was relatively small. Thirdly, the selection of the controls varied among studies. Some studies used a healthy population as controls, while others used hospitalized patients without HBV infection as controls; therefore, it is possible that selection bias existed, since these studies may have included controls at different risks of developing DM. Furthermore, heterogeneity was significant across studies, which decreased the reliability of the summary OR estimates. Finally, data were only obtained from papers published in English or Chinese, which could have also contributed to selection bias.

In conclusion, the present study indicates that patients with HBV infection are at higher risk of developing DM compared with uninfected patients and that $\mathrm{HBV}$ infection may be a potential risk factor for DM development. Due to the considerable heterogeneity across the studies and the limitations of this meta-analysis, further research is required to investigate the possible association between HBV infection and the prevalence of DM. 


\section{Acknowledgements}

This study was supported by the Natural Science Foundation of Guangdong (grant no. S2013010016631) and the Guangdong Supporting Grant for Outstanding Talent (grant no. C1030925).

\section{References}

1. Shaw JE, Sicree RA and Zimmet PZ: Global estimates of the prevalence of diabetes for 2010 and 2030. Diabetes Res Clin Pract 87: 4-14, 2010.

2. Lee JY, Jeon I, Lee JM, et al: Diabetes mellitus as an independent risk factor for lung cancer: A meta-analysis of observational studies. Eur J Cancer 49: 2411-2423, 2013.

3. Ott JJ, Stevens GA, Groeger J and Wiersma ST: Global epidemiology of hepatitis B virus infection: New estimates of age-specific HBsAg seroprevalence and endemicity. Vaccine 30 2212-2219, 2012

4. Keyvani H, Agah S, Kabir A and Alavian SM: Prevalence and risk factors of isolated anti-HBc antibody and occult hepatitis B infection in hemodialysis patients: A nationwide study. Ann Hepatol 12: 213-219, 2013.

5. Schillie SF, Xing J, Murphy TV and Hu DJ: Prevalence of hepatitis B virus infection among persons with diagnosed diabetes mellitus in the United States, 1999-2010. J Viral Hepat 19: 674-676, 2012.

6. Li-Ng M, Tropp S, Danoff A and Bini EJ: Association between chronic hepatitis B virus infection and diabetes among Asian Americans and Pacific Islanders. Dig Liver Dis 39: 549-556, 2007.

7. Yang SQ, Chen HS, Jiang D, et al: Relationship between chronic hepatitis $\mathrm{C}$ and type II diabetes mellitus. Zhonghua Shi Yan He Lin Chuang Bing Du Xue Za Zhi 17: 46-49, 2003 (In Chinese).

8. Qureshi H, Ahsan T, Mujeeb SA, et al: Diabetes mellitus is equally frequent in chronic HCV and HBV infection. J Pak Med Assoc 52: 280-283, 2002

9. Yang JH, Sun CM, Xu XB and Bao CX: Susceptibility of diabetes in patients with positive hepatitis B surface antigen. Zhongguo Wuzhen Xue Zazhi 2: 564, 2002 (In Chinese).

10. Spradling PR, Simons B, Narayanan M, et al: Incidence of diabetes mellitus in a population-based cohort of persons with chronic hepatitis B virus infection. J Viral Hepat 20: 510-513, 2013.

11. Jan CF, Chen CJ, Chiu YH, et al: A population-based study investigating the association between metabolic syndrome and hepatitis $\mathrm{B} / \mathrm{C}$ infection (Keelung Community-based Integrated Screening study No. 10). Int J Obes (Lond) 30: 794-799, 2006.

12. Reilly ML, Schillie SF, Smith E, et al: Increased risk of acute hepatitis B among adults with diagnosed diabetes mellitus. J Diabetes Sci Technol 6: 858-866, 2012.

13. Huang ZS, Huang TS, Wu TH, et al: Asymptomatic chronic hepatitis B virus infection does not increase the risk of diabetes mellitus: A ten-year observation. J Gastroenterol Hepatol 25: 1420-1425, 2010.

14. Wang CS, Chang TT, Yao WJ, et al: The impact of smoking on incident type 2 diabetes in a cohort with hepatitis B but not hepatitis C infection. J Viral Hepat: Aug. 31, 2010 (Epub ahead of print).
15. Imazeki F, Yokosuka O, Fukai K, et al: Prevalence of diabetes mellitus and insulin resistance in patients with chronic hepatitis C: Comparison with hepatitis B virus-infected and hepatitis C virus-cleared patients. Liver Int 28: 355-362, 2008.

16. Zhao P, Wang JB and Jiao J: Investigation on the incidence of diabetes in chronic hepatitis $\mathrm{C}$ patients and their $\mathrm{HCV}$ genotypes. Zhonghua Gan Zang Bing Za Zhi 14: 86-88, 2006 (In Chinese).

17. Knobler H, Schihmanter R, Zifroni A, et al: Increased risk of type 2 diabetes in noncirrhotic patients with chronic hepatitis $\mathrm{C}$ virus infection. Mayo Clin Proc 75: 355-359, 2000.

18. Naing C, Mak JW, Ahmed SI and Maung M: Relationship between hepatitis $\mathrm{C}$ virus infection and type 2 diabetes mellitus: Meta-analysis. World J Gastroenterol 18: 1642-1651, 2012.

19. Picardi A, D'Avola D, Gentilucci UV, et al: Diabetes in chronic liver disease: From old concepts to new evidence. Diabetes Metab Res Rev 22: 274-283, 2006.

20. Harrison SA: Liver disease in patients with diabetes mellitus. J Clin Gastroenterol 40: 68-76, 2006.

21. Raddatz D and Ramadori G: Carbohydrate metabolism and the liver: Actual aspects from physiology and disease. Z Gastroenterol 45: 51-62, 2007.

22. Tappy L and Minehira K: New data and new concepts on the role of the liver in glucose homeostasis. Curr Opin Clin Nutr Metab Care 4: 273-277, 2001.

23. Teli T, Xanthaki D and Karalis KP: Regulation of appetite and insulin signaling in inflammatory states. Ann NY Acad Sci 1083: 319-328, 2006.

24. Postic C, Dentin R and Girard J: Role of the liver in the control of carbohydrate and lipid homeostasis. Diabetes Metab 30: 398-408, 2004

25. Collier B, Dossett LA, May AK and Diaz JJ: Glucose control and the inflammatory response. Nutr Clin Pract 23: 3-15, 2008.

26. Leclercq IA, Da Silva Morais A, Schroyen B, et al: Insulin resistance in hepatocytes and sinusoidal liver cells: Mechanisms and consequences. J Hepatol 47: 142-156, 2007.

27. Majano PL, García-Monzón C, López-Cabrera M, et al: Inducible nitric oxide synthase expression in chronic viral hepatitis. Evidence for a virus-induced gene upregulation. J Clin Invest 101: 1343-1352, 1998.

28. Yoffe B, Burns DK, Bhatt HS and Combes B: Extrahepatic hepatitis B virus DNA sequences in patients with acute hepatitis B infection. Hepatology 12: 187-192, 1990.

29. Shimoda T, Shikata T, Karasawa T, et al: Light microscopic localization of hepatitis B virus antigens in the human pancreas. Possibility of multiplication of hepatitis B virus in the human pancreas. Gastroenterology 81: 998-1005, 1981.

30. Ji D, Cheng J, Dong Z, et al: Screening and identification of genes trans-regulated by HBV pre-S2 protein with cDNA microarray. World Chin J Digestology 12: 1559-1563, 2004 (In Chinese)

31. Selvin E, Parrinello CM, Sacks DB, et al: Trends in prevalence and control of diabetes in the United States, 1988-1994 and 1999-2010. Ann Intern Med 160: 517-525, 2014.

32. Guariguata L, Whiting DR, Hambleton I, et al: Global estimates of diabetes prevalence for 2013 and projections for 2035. Diabetes Res Clin Pract 103: 137-149, 2014. 